\title{
Axisymmetric non-Newtonian drops treated with a boundary integral method
}

\author{
E.M. TOOSE ${ }^{1}$, D. VAN DEN ENDE ${ }^{1}$, B.J. GEURTS ${ }^{2}$, J.G.M. KUERTEN ${ }^{2}$ and \\ P.J. ZANDBERGEN ${ }^{2}$ \\ ${ }^{1}$ Rheology Group, Department of Applied Physics, ${ }^{2}$ Department of Applied Mathematics, University of Twente, \\ P.O. Box 217, 7500 AE Enschede, The Netherlands
}

Received 27 April 1995

\begin{abstract}
A boundary integral method for the simulation of the time-dependent deformation of axisymmetric Newtonian or non-Newtonian drops suspended in a Newtonian fluid subjected to an axisymmetric flow field is developed. The boundary integral formulation for Stokes flow is used and the non-Newtonian stress is treated as a source term which yields an extra integral over the domain of the drop. By transforming the integral representation for the velocity to cylindrical coordinates we can reduce the dimension of the computational problem. The integral equation for the velocity remains of the same form as in Cartesian coordinates, and the Green's functions are transformed explicitly to cylindrical coordinates. Besides a numerical validation of the method we present simulation results for a Newtonian drop and a drop consisting of an Oldroyd-B fluid. The results for the Newtonian drop are consistent with results from the literature. The deformation process of the non-Newtonian drop for small capillary numbers appears to be governed by two relaxation times.
\end{abstract}

\section{Introduction}

The deformation of viscous drops immersed in a Newtonian fluid, due to an external linear shear, is a subject of considerable experimental and theoretical research (see e.g. Rallison [1] and Stone [2] for a survey). If the drops are sufficiently small, the dynamics of these drops can be analyzed through an investigation of the Stokes equations since the corresponding Reynolds number is small enough to justify the neglect of inertia forces. Most theoretical work has been directed towards the case that the drop consists of a second Newtonian fluid in which the interface between the drop and the surrounding fluid is considered infinitely thin and characterized by a constant surface tension. The velocity field can, in these cases, be expressed in terms of a boundary integral over the surface of the drop [3]. In this paper we extend this boundary integral approach to the case of an axisymmetric non-Newtonian drop in an axisymmetric external flow. Due to the non-Newtonian contribution to the stress tensor, a domain integral over the drop appears in the expression for the velocity field, next to a boundary integral term. Through an explicit integration over the azimuthal direction, an efficient and accurate numerical method results with which the temporal and steady state deformation of the drop can be simulated.

The interest in the dynamics of viscous drops arises mainly from a desire to understand the rheology of emulsions, the mechanisms of heterogeneous mixing and the deformation of biological cells [1]. The mathematical basis for the theoretical work related to these different fields of application can be found in the pioneering work of H.A. Lorentz [4]. Lorentz was the first to derive, in essence, the boundary integral expression for the velocity around a drop at low Reynolds numbers, which is of key importance to all theoretical investigations in this field. The diversity of the many subsequent developments which, to some extent, can 
be seen as originating from this work, would probably have surprised Lorentz whose interest in applied physics was aroused only late in his life in relation to the construction of the 'Zuiderzeewerken' [5].

The deformation of neutrally buoyant Newtonian drops in viscous extensional flows at low Reynolds number was first studied numerically by Youngren [6], using a boundary element method. This method has the advantage of reducing the dimension of the computational problem, which significantly reduces the computational cost. The method has been used extensively by others as well in order to simulate the behavior of Newtonian drops in simple flow fields. Apart from improvements on the numerical method (Huang [7]), recent studies have tackled more complicated flow problems (e.g. breakup of drops, Tjahjadi [8]) and incorporated additional physical phenonema (e.g. the effects due to surfactants, Stone [9] and the elasticity of the membrane, $\mathrm{Li}[10]$ ).

In many applications, however, the drop consists of a non-Newtonian fluid and the development of the boundary integral method is much more complicated due to the domain integral which arises from the non-Newtonian contributions. This implies the introduction of a grid covering the volume of the drop, next to the definition of discrete points on the boundary, and adds considerably to the numerical cost of simulations of non-Newtonian drops. However, compared to a more direct (finite difference) discretization of the Stokes equations, which would even require a grid covering the much larger region exterior to the drop, the boundary integral method for non-Newtonian drops is more efficient. Moreover, in several applications the region containing a non-Newtonian fluid forms only a small portion of the flow-problem, e.g. a vesicle in which the drop is formed by a Newtonian fluid which is encapsulated by a non-Newtonian lipid bilayer [11], and the boundary integral method can be used effectively. Bush [12], [13] adopted the boundary element method to analyze extrusion experiments with non-Newtonian fluids. The extension to non-Newtonian drops immersed in a Newtonian fluid in axisymmetric flow is considered in the present paper. This extension is valid provided certain assumptions on the smoothness of the non-Newtonian stress tensor are satisfied. These are verified numerically for a two-dimensional drop [14] and in the present axisymmetric flow in case the drop contains an Oldroyd-B fluid. The method is illustrated by comparing simulation results for Newtonian drops with results from literature. Moreover, we consider the non-Newtonian behavior of the drop and investigate the dominant relaxation times associated with the temporal deformation.

The organization of this paper is as follows. In section 2 we present the governing equations and express the velocity field in terms of a domain integral involving the non-Newtonian stress tensor and a boundary integral arising from the Newtonian contributions. Section 3 is devoted to the specification of the expression for the velocity field in axisymmetric flow. Through an explicit integration over the azimuthal direction, the corresponding Green's functions for this flow are obtained. The numerical method used to simulate the deformation of the drop is described in section 4 and simulation results for both Newtonian and non-Newtonian drops, consisting of an Oldroyd-B fluid, are presented in section 5. Finally, we summarize our findings in section 6. 


\section{Governing equations}

We consider an isotropic non-Newtonian drop placed in an unbounded Newtonian fluid with viscosity $\eta$, subjected to a linear elongational flow. This velocity field is defined by:

$$
u_{1}=G x_{1}, \quad u_{2}=-\frac{1}{2} G x_{2}, \quad u_{3}=-\frac{1}{2} G x_{3},
$$

with $u_{j}$ the $j$-th component of the velocity field with respect to a Cartesian coordinate frame $\left\{e_{1}, e_{2}, e_{3}\right\}$ and $G$ the magnitude of the flow. The interfacial tension acting between the two fluids is denoted by $\sigma$ and is assumed to be constant along the interface. The radius of the (spherical) drop in a quiescent fluid flow is denoted by $a$. The fluid is incompressible and buoyancy is considered to be absent, i.e. we assume that the densities of the two fluids are equal. Throughout we will work with dimensionless variables: all lengths are scaled with the undeformed capsule radius $a$, velocities by $a G$ and viscosities by $\eta$ (Li [10]). In order to characterize the degree of distortion, one commonly defines a deformation parameter $D$ as:

$$
D=\frac{\ell_{\max }-\ell_{\min }}{\ell_{\max }+\ell_{\min }}
$$

where $\ell_{\max }$ and $\ell_{\min }$ denote the longest and shortest lengths in the deformed state respectively. From analytical studies of a spherical drop containing a Newtonian fluid (Cox [15]) it appeared that the deformation can be characterized by two parameters:

$$
C=\frac{\eta G a}{\sigma} \text { and } \lambda=\frac{\eta^{(i)}}{\eta}
$$

where $C$ is the capillary number which, is a measure of the ratio between the viscous and interfacial tension stresses and $\lambda$ is the ratio between the interior viscosity $\eta^{(i)}$ and exterior viscosity $\eta$.

Assuming that the Reynolds number $\left(\operatorname{Re}=\rho a^{2} G / \eta\right.$, with $\rho$ the density) is small we can describe the fluid motion by the Stokes equations:

$$
\begin{aligned}
\partial_{j} \pi_{i j} & =0, \quad \forall \boldsymbol{x} \notin \partial \Omega \text { and } i=1, \ldots, 3 \\
\partial_{j} u_{j} & =0, \quad \forall \boldsymbol{x} \notin \partial \Omega,
\end{aligned}
$$

with $\partial_{j}=\partial / \partial x_{j}$ and $\partial \Omega$ the boundary between the internal domain $\Omega^{(i)}$ and the external domain $\Omega^{(o)}$. In $(2.4,2.5)$, as in the remainder of this paper, the summation convention is used to indicate summation over repeated indices. The total stress tensor $\pi_{i j}$ is defined as:

$$
\pi_{i j}= \begin{cases}-P \delta_{i j}+\lambda \dot{\gamma}_{i j}+\tau_{i j}^{N N} & \forall x \in \Omega^{(i)} \\ -P \delta_{i j}+\dot{\gamma}_{i j} & \forall x \in \Omega^{(o)},\end{cases}
$$

where $\dot{\gamma}_{i j}=\partial_{i} u_{j}+\partial_{j} u_{i}$ is the rate-of-strain tensor, $P$ the isotropic pressure and $\tau_{i j}^{N N}$ the nonNewtonian stress tensor which satisfies a certain constitutive equation. In this paper we restrict ourselves to the Maxwell model which finds its origin in polymer rheology and contains two parameters: a relaxation time $\mu$ and the polymer contribution to the zero-shear-rate viscosity $\eta^{(p)}$. The constitutive equation of this model is given by:

$$
\mathcal{D}_{t} \tau_{i j}^{N N}+\frac{1}{D e} \tau_{i j}^{N N}=\frac{\lambda^{(p)}}{D e} \dot{\gamma}_{i j}
$$


where $\mathcal{D}_{t}$ is the upper-convected time derivative, $D e=G \mu$ the Deborah or Weissenberg number and $\lambda^{(p)}=\eta^{(p)} / \eta$. The upper-convected time derivative is defined as (Bird [17]):

$$
\mathcal{D}_{t} \tau_{i j}=\mathrm{d}_{t} \tau_{i j}-\tau_{i k} \partial_{k} u_{j}-\partial_{k} u_{i} \tau_{k j},
$$

with $\mathrm{d}_{t} \tau_{i j}$ the material time derivative. Since we can always incorporate a part of the Newtonian stress tensor in the non-Newtonian stress tensor, we can put $\lambda=1$ in (2.6) without loss of generality [14]. This makes it possible to study Newtonian drops in a surrounding Newtonian fluid with different viscosity by setting the 'non-Newtonian' stress tensor equal to:

$$
\tau_{i j}^{N N}=(\lambda-1) \dot{\gamma}_{i j} \text {. }
$$

The flow field satisfies the following matching and asymptotic conditions:

$$
\begin{gathered}
{\left[u_{i}\right]_{\partial \Omega}=0, C\left[\pi_{i j} n_{j}\right]_{\partial \Omega}=k n_{i}} \\
u_{i}=u_{i}^{\infty}, \text { as }|\boldsymbol{x}| \rightarrow \infty,
\end{gathered}
$$

where $[\cdot]_{\partial \Omega}$ denotes the discontinuity of the quantity between the brackets across the surface of the drop from the outside to the inside, $\boldsymbol{n}$ is the outward unit normal and $k\left(=\partial_{j} n_{j}\right)$ is the boundary curvature. In addition, there is a kinematic constraint, which requires that a fluid element on the surface $\partial \Omega$ remains on the surface for all time. This kinematic constraint may be expressed by an evolution equation:

$$
\mathrm{d}_{t} x_{i}=u_{i}, \quad \forall \boldsymbol{x} \in \partial \Omega(t) .
$$

For both evolution Eqs. (2.7) and (2.12) initial conditions need to be specified. For the interface $\partial \Omega(0)$ we start with a spherical shape, whereas for the non-Newtonian contribution to the stress tensor we assume an isotropic stress distribution, (i.e. $\tau_{i j}^{N N}(0)=Q \delta_{i j}$, with $Q$ an arbitrary constant).

Assuming that the non-Newtonian stress tensor is known at time $t$ we can construct a solution for the velocity by means of boundary integral equations. Following Ladyzhenskaya [3] it can be derived that the integral representation for the velocity is given by (Toose [14]):

$$
u_{k}(\boldsymbol{x})=u_{k}^{\infty}(\boldsymbol{x})-\int_{\Omega^{(i)}} \tau_{i j}^{N N}(\boldsymbol{y}) \partial_{j} J_{i k}(\boldsymbol{r}) \mathrm{d} \boldsymbol{y}-\frac{1}{C} \int_{\partial \Omega} J_{i k}(\boldsymbol{r}) k(\boldsymbol{y}) n_{i}(\boldsymbol{y}) \mathrm{d} S_{y},
$$

with $\boldsymbol{r}=\boldsymbol{x}-\boldsymbol{y}$. The kernel $J_{i k}(\boldsymbol{r})$ is called the Green's function for the Stokes problem, and is given by (Lorentz [16] page 32, Ladyzheskaya [3]):

$$
J_{i k}(r)=\frac{1}{8 \pi}\left\{\frac{\delta_{i k}}{|r|}+\frac{r_{i} r_{k}}{|r|^{3}}\right\} \text {. }
$$

In the derivation of (2.13) we have used the Gauss divergence theorem to rewrite the volume integral. However, in order to apply the divergence theorem it is necessary that the nonNewtonian stress tensor is continuous and has bounded derivatives. Numerical simulations of a two-dimensional drop in [14] have shown that the stress tensor apparently satisfies these conditions for all time.

With expression (2.13) we have found the solution for the velocity field in the entire flow domain, provided that the non-Newtonian stress tensor and the shape of the surface $\partial \Omega$ are given. By calculating $u$ and subsequently updating $\partial \Omega$ and $\tau_{i j}^{N N}$ through Eqs. (2.7) and (2.12) the evolution of both $\partial \Omega$ and $\tau_{i j}^{N N}$ can be simulated. In the next section we will transform the 


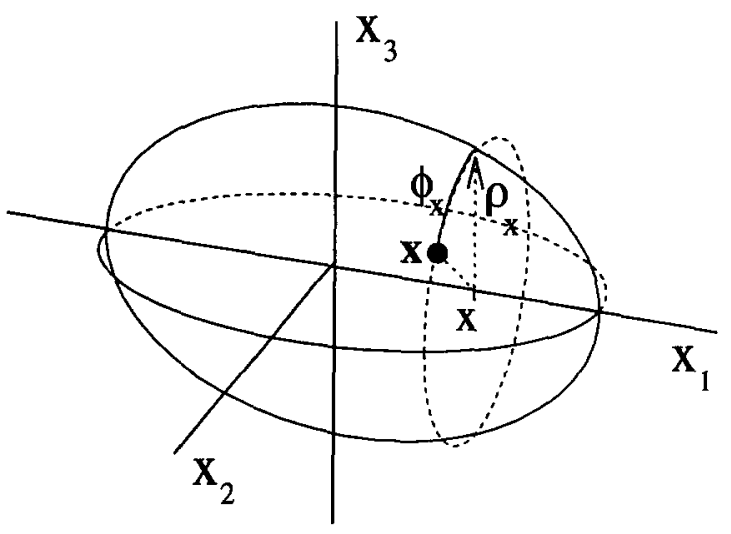

Fig. 1. Schematic illustration of the cylindrical coordinate system $\left(x, \rho_{x}, \phi_{x}\right)$.

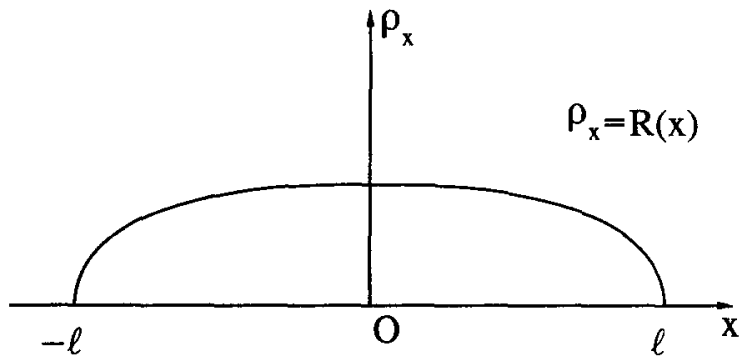

Fig. 2. The function $\rho_{x}=R(x)$ describing the drop surface.

integral equation to cylindrical coordinates in order to reduce the dimension of the problem through an explicit integration over the angle of revolution.

\section{Axisymmetric Stokes flow}

In this section we consider the case in which the imposed flow $\boldsymbol{u}^{\infty}$ is axisymmetric around the $x_{1}$ axis and has no swirling motion. The initial shape of the capsule is also assumed to be axisymmetric around the $x_{1}$ axis. By transforming the integral Eq. (2.13) to cylindrical coordinates and performing the integration in the azimuthal direction analytically we can reduce the dimension of the computational problem. In subsection 3.1 we introduce cylindrical coordinates and transform Eq. (2.13). In subsection 3.2 we perform the integration in the azimuthal direction for the boundary integral term and the volume integral term separately.

\subsection{GREEN'S FUNCTIONS FOR AXISYMMETRIC STOKES FLOW}

In this subsection the integral representation for an axisymmetric drop placed in an axisymmetric flow is derived. It is convenient to use cylindrical coordinates defined as:

$$
\left\{\begin{array}{l}
x_{1}=x \\
x_{2}=\rho_{x} \cos \left(\phi_{x}\right) \\
x_{3}=\rho_{x} \sin \left(\phi_{x}\right)
\end{array}\right.
$$


where $x, \rho_{x}$ and $\phi_{x}$ indicate the axial, radial and azimuthal components respectively. The subscript $x$ indicates that $\rho_{x}$ and $\phi_{x}$ are defined with reference to the point $\boldsymbol{x}$ (see Fig. 1). Using these coordinates we can describe the surface $\partial \Omega$ as:

$$
\rho_{x}=R(x) \text { for }-\ell \leq x \leq \ell,
$$

with $R(-x)=R(x)$ and $R(\ell)=0$, as shown in Fig. 2. In the sequel we denote components with respect to cylindrical coordinates with an over-bar.

To perform the transformation, it will be convenient to use a natural base $\left\{\boldsymbol{g}_{x}, \boldsymbol{g}_{\rho}, \boldsymbol{g}_{\phi}\right\}$ for the coordinates $\left(x, \rho_{x}, \phi_{x}\right)$ which is related to the orthonormal base $\left\{\boldsymbol{\delta}_{x}, \boldsymbol{\delta}_{\rho}, \delta_{\phi}\right\}$ through (Bird [17]):

$$
\boldsymbol{g}_{x}=\boldsymbol{\delta}_{x} ; \quad \boldsymbol{g}_{\rho}=\boldsymbol{\delta}_{\rho} ; \quad \boldsymbol{g}_{\phi}=\rho_{x} \boldsymbol{\delta}_{\phi}
$$

Similarly, we can define a set of reciprocal base vectors:

$$
g^{x}=\delta_{x} ; \quad g^{\rho}=\delta_{\rho} ; \quad g^{\phi}=\frac{1}{\rho_{x}} \delta_{\phi}
$$

A vector $u$ can be represented as:

$$
\boldsymbol{u}=u_{k} \boldsymbol{e}_{k}=\bar{u}^{\alpha} \boldsymbol{g}_{\alpha}=\bar{u}_{\alpha} \boldsymbol{g}^{\alpha},
$$

in which $\bar{u}_{\alpha}$ and $\bar{u}^{\alpha}$ are referred to as the covariant and contravariant components of the vector $u$. In Eq. (3.5) summation is performed over repeated lower and higher indices. In the sequel we use the covariant representation for the velocity and stresses. Using (3.1) and (3.5) we find that the transformation of a velocity component $u_{i}$ and a stress component $\tau_{i j}$ from cylindrical to Cartesian coordinates is given by:

$$
u_{i}(\boldsymbol{x})=\frac{\partial \bar{x}^{\alpha}}{\partial x^{i}} \bar{u}_{\alpha}(\overline{\boldsymbol{x}}) ; \quad \tau_{i j}(\boldsymbol{x})=\frac{\partial \bar{x}^{\alpha}}{\partial x^{i}} \frac{\partial \bar{x}^{\beta}}{\partial x^{j}} \bar{\tau}_{\alpha \beta}(\overline{\boldsymbol{x}}),
$$

respectively. Using these transformation formulas we can write (2.13) as:

$$
\bar{u}_{\alpha}(\overline{\boldsymbol{x}})=\bar{u}_{\alpha}^{\infty}(\overline{\boldsymbol{x}})-\int_{\bar{\Omega}^{(i)}} \bar{V}_{\alpha}^{\gamma \beta}(\overline{\boldsymbol{x}} ; \overline{\boldsymbol{y}}) \bar{\tau}_{\beta \gamma}^{N N}(\overline{\boldsymbol{y}})|Q(\overline{\boldsymbol{y}})| \mathrm{d} \overline{\boldsymbol{y}}-\frac{1}{C} \int_{\partial \bar{\Omega}} \bar{J}_{\alpha}^{\beta}(\overline{\boldsymbol{x}} ; \overline{\boldsymbol{y}}) \bar{n}_{\beta}(\overline{\boldsymbol{y}}) k(\overline{\boldsymbol{y}}) \mathrm{d} \bar{S}_{y},
$$

with $\bar{\Omega}^{(i)}$ the transformed internal domain, $\partial \bar{\Omega}$ the corresponding boundary and $|Q(\bar{y})|=\rho_{y}$ the Jacobian. The kernels $\bar{V}_{\alpha}^{\gamma \beta}(\bar{x} ; \bar{y})$ and $\bar{J}_{\alpha}^{\beta}(\bar{x} ; \bar{y})$ are given by:

$$
\begin{aligned}
& \bar{V}_{\alpha}^{\gamma \beta}(\overline{\boldsymbol{x}} ; \overline{\boldsymbol{y}})=\frac{\partial x^{k}}{\partial \bar{x}^{\alpha}} \frac{\partial \bar{y}^{\beta}}{\partial y^{i}} \frac{\partial \bar{y}^{\gamma}}{\partial y^{j}} \partial_{j} J_{i k}(\overline{\boldsymbol{x}}-\overline{\boldsymbol{y}}) \\
& \bar{J}_{\alpha}^{\beta}(\overline{\boldsymbol{x}} ; \overline{\boldsymbol{y}})=\frac{\partial x^{k}}{\partial \bar{x}^{\alpha}} \frac{\partial \bar{y}^{\beta}}{\partial y^{i}} J_{i k}(\overline{\boldsymbol{x}}-\overline{\boldsymbol{y}}),
\end{aligned}
$$

where we have expressed the Green's function $J_{i k}$ in cylindrical coordinates by using the transformation formulas in (3.1). Expression (3.7) is the integral representation for the velocity in cylindrical coordinates and the kernels $\bar{J}_{\alpha}^{\beta}(\bar{x} ; \bar{y})$, and $\bar{V}_{\alpha}^{\gamma \beta}(\bar{x} ; \bar{y})$ are the corresponding Green's functions. 


\subsection{NON-NEWTONIAN DROPS IN AXISYMMETRIC FLOW}

In this section the integral Eq. (3.7) is simplified by performing the integration over the azimuthal direction analytically. First, we study the integral equation of a Newtonian drop in an axisymmetric flow. Using the fact that for an axisymmetric drop the azimuthal component of the unit normal is zero and the curvature is independent of the azimuthal coordinate we can write the velocity as:

$$
\bar{u}_{\alpha}(\overline{\boldsymbol{x}})=\bar{u}_{\alpha}^{\infty}(\overline{\boldsymbol{x}})-\frac{1}{C} \int_{L} k(y) \bar{n}_{\beta}(y) R(y) \int_{0}^{2 \pi} \bar{J}_{\alpha}^{\beta}(\overline{\boldsymbol{x}} ; \overline{\boldsymbol{y}}) \mathrm{d} \phi_{y} \mathrm{~d} \ell_{y}
$$

where $R(y) \mathrm{d} \phi_{y} \mathrm{~d} \ell_{y}$ is the unit surface area with $\mathrm{d} \ell_{y}=\left\{1+\left(\partial_{y} R(y)\right)^{2}\right\}^{1 / 2} \mathrm{~d} y$ and $L$ the intersection of the surface described by $\rho_{x}=R(x)$ and the plane $\phi_{x}=0$. The integral in the $\phi$ direction is given by:

$$
\bar{M}_{\alpha}^{\beta}(\overline{\boldsymbol{x}} ; y)=\int_{0}^{2 \pi} \bar{J}_{\alpha}^{\beta}(\overline{\boldsymbol{x}} ; \overline{\boldsymbol{y}}) \mathrm{d} \phi_{y} .
$$

Using the expression for the Green's function (3.9) and the transformation formulas in (3.1), we can express $\bar{M}_{\alpha}^{\beta}(x ; y)$ in terms of complete elliptic integrals of the first and second kind [18] (see Appendix). These integrals can be computed with an iterative method. Since the constitutive equation for a Newtonian fluid obeys material frame indifference (translational and rotational invariance), the resulting flow is axisymmetric when the drop interface $\partial \Omega$ and $\boldsymbol{u}^{\infty}$ are axisymmetric (Schowalter [19]). Hence, $\bar{u}_{3}(\overline{\boldsymbol{x}})=0$, since the applied velocity field has no swirling motion. This property also follows directly from Eq. (3.10), since $\bar{M}_{\alpha}^{\beta}(x ; y)$ obeys (see Appendix):

$$
\bar{M}_{3}^{\beta} \bar{n}_{\beta}=\bar{M}_{3}^{3} \bar{n}_{3}=0,
$$

which implies that the contribution of the boundary integral to the azimuthal component of the velocity is zero. Using this, we can rewrite (3.10) as:

$$
\left\{\begin{array}{l}
\bar{u}_{\alpha}(\overline{\boldsymbol{x}})=\bar{u}_{\alpha}^{\infty}(\overline{\boldsymbol{x}})-\frac{1}{C} \int_{L} k(y) \bar{n}_{\beta}(y) \bar{M}_{\alpha}^{\beta}(\overline{\boldsymbol{x}} ; y) R(y) \mathrm{d} \ell_{y}, \quad \alpha=1,2 \\
\bar{u}_{3}(\overline{\boldsymbol{x}})=0 .
\end{array}\right.
$$

which is a line integral in a two dimensional space. Hence, for the Newtonian drop the main problem is reduced to the evaluation of a one dimensional integral.

In the case of a non-Newtonian drop, the contribution of the non-Newtonian stress tensor to the velocity is given by:

$$
I_{\alpha}=\int_{\bar{\Omega}^{(i)}} \bar{V}_{\alpha}^{\gamma \beta}(\overline{\boldsymbol{x}} ; \overline{\boldsymbol{y}}) \bar{\tau}_{\beta \gamma}^{N N}(\overline{\boldsymbol{y}})|Q(\overline{\boldsymbol{y}})| \mathrm{d} \overline{\boldsymbol{y}}=\int_{\bar{\Omega}^{(i)}} \bar{V}_{\alpha}^{\gamma \beta}(\overline{\boldsymbol{x}} ; \overline{\boldsymbol{y}}) \bar{\tau}_{\beta \gamma}^{N N}(\overline{\boldsymbol{y}}) \rho_{y} \mathrm{~d} \phi_{y} \mathrm{~d} \rho_{y} \mathrm{~d} y .
$$

To perform the integration over the azimuthal direction analytically, we assume that the non-Newtonian stress tensor satisfies the following conditions:

1. $\bar{\tau}_{\beta \gamma}^{N N}(\overline{\boldsymbol{y}})=\bar{\tau}_{\gamma \beta}^{N N}(\overline{\boldsymbol{y}})$ (symmetric)

2. $\bar{\tau}_{13}^{N N}(\overline{\boldsymbol{y}})=\bar{\tau}_{23}^{N N}(\overline{\boldsymbol{y}})=0$

3. $\bar{\tau}_{\beta \gamma}^{N N}(\overline{\boldsymbol{y}})$ is independent of $\phi_{y}$.

The physical interpretation of these conditions is as follows. The first condition implies that no electric or magnetic dipoles are present. The second condition states that the constitutive 
equation for the non-Newtonian stress tensor obeys material frame invariance. The last condition follows directly from the first two and the fact that the non-Newtonian fluid is isotropic. These conditions also imply that the non-Newtonian stress tensor has no contribution to the azimuthal velocity component. Analyzing the Maxwell model (2.7) we find that this model satisfies all these conditions. Hence, we can rewrite (3.13) as:

$$
I_{\alpha}=\int_{\bar{S}^{(\phi)}} \bar{\tau}_{\beta \gamma}^{N N}\left(y, \rho_{y}\right) \bar{W}_{\alpha}^{\gamma \beta}\left(\bar{x} ; y, \rho_{y}\right) \rho_{y} \mathrm{~d} \rho_{y} \mathrm{~d} y
$$

where $\bar{S}^{(\phi)}$ is part of the plane $\phi_{x}=0$ which is contained in $\bar{\Omega}^{(i)}$, (see Fig. 2). The kernel $\bar{W}_{\alpha}^{\gamma \beta}\left(\bar{x} ; y, \rho_{y}\right)$ is given by:

$$
\bar{W}_{\alpha}^{\gamma \beta}\left(\overline{\boldsymbol{x}}, y, \rho_{y}\right)=\int_{0}^{2 \pi} \bar{V}_{\alpha}^{\gamma \beta}(\overline{\boldsymbol{x}} ; \overline{\boldsymbol{y}}) \mathrm{d} \phi_{y},
$$

which can be expressed in terms of complete elliptic integrals of the first and second kind with use of the expression for the Green's function (3.8) and the transformation formulas in (3.1) (see Appendix). Using the expressions for (3.16) given in the Appendix we find that $\bar{W}_{\alpha}^{\gamma \beta}\left(\overline{\boldsymbol{x}}, y, \rho_{y}\right)$ obeys:

$$
\bar{\tau}_{\beta \gamma}^{N N} \bar{W}_{3}^{\gamma \beta}=0 \text {, }
$$

which implies that $I_{3}=0$. This confirms that the non-Newtonian stress tensor gives no contribution to the velocity in the azimuthal direction. Combining (3.12) and (3.17), we obtain the following expression for the velocity field:

$$
\begin{cases}\bar{u}_{\alpha}(\overline{\boldsymbol{x}})=\bar{u}_{\alpha}^{\infty}(\overline{\boldsymbol{x}}) & -\int_{S^{(\phi)}} \bar{\tau}_{\beta \gamma}^{N N}\left(y, \rho_{y}\right) \bar{W}_{\alpha}^{\gamma \beta}\left(\overline{\boldsymbol{x}} ; y, \rho_{y}\right) \rho_{y} \mathrm{~d} \rho_{y} \mathrm{~d} y \\ & -\frac{1}{C} \int_{L} k(y) \bar{n}_{\beta}(y) \bar{M}_{\alpha}^{\beta}(\overline{\boldsymbol{x}} ; y) \rho_{y} \mathrm{~d} \ell_{y}, \quad \alpha=1,2 \\ \bar{u}_{3}(\overline{\boldsymbol{x}})=0, & \end{cases}
$$

which is a surface integral in two dimensions. With expression (3.18) we have reduced the three-dimensional problem to a two-dimensional one. The presence of the non-Newtonian stress, however, makes it impossible to reduce the problem to one dimension, as was possible for the Newtonian drop. This implies that the computational effort required to solve non-Newtonian problems is considerably greater than for the corresponding Newtonian problems.

With expression (3.18) we have found the solution for the velocity field in the entire flow domain, provided that the non-Newtonian stress tensor and the shape of the interface are given. In the next section we will discuss a method for solving the full time-dependent problem and provide an algorithm to calculate $\bar{\tau}_{\beta \gamma}^{N N}$.

\section{Numerical procedure}

In this section the numerical procedure used to evaluate the boundary and domain integrals, the non-Newtonian stress tensor and the shape of the surface $\partial \Omega$ are given in detail. In subsection 4.1 algorithms are presented to simulate the evolution of a non-Newtonian and Newtonian axisymmetric drop, respectively. The numerical method to calculate the velocity field is described in subsection 4.2. In subsection 4.3 the time integration of the evolution Eqs. (2.7) and (2.12) is presented. 


\subsection{NUMERICAL ALGORITHMS}

The complete procedure used to simulate the evolution of the non-Newtonian axisymmetric drop can be sketched as follows. Suppose the stress tensor, $\bar{\tau}_{\beta \gamma}^{N N}$, the velocity field and the shape of $\partial \Omega$, and hence $L$, are given at time $t$. Then we can use (2.7) to calculate the nonNewtonian stress tensor at $t+\Delta t$, whereas the new shape of the drop at $t+\Delta t$ can be obtained using (2.12). With this new stress tensor and shape, a new velocity field at time level $t+\Delta t$ can be calculated using (3.18). Repeating this explicit time integration procedure gives the evolution of the stress tensor, the velocity field and the boundary shape $L$. After some initializations (i.e. $\bar{\tau}_{\beta \gamma}^{N N}=0$, the initialization of the shape of the boundary and the calculation of the initial velocity field using (3.18) with $\bar{\tau}_{\beta \gamma}^{N N}(0)$ and $L(0)$ ), several steps have to be taken at every time step. These are summarized in the following algorithm:

\section{ALGORITHM 4.1.: non-Newtonian fluids}

1. update the shape of the boundary by solving (2.12) to yield $L(t+\Delta t)$,

2. calculate the stress tensor at $t+\Delta t$ by solving (2.7) given $\bar{u}_{\alpha}$ at time $t$,

3. calculate the velocity field at $t+\Delta t$ using (3.18) given $\bar{\tau}_{\beta \gamma}^{N N}$ and $L$ from step 1 and 2 .

The procedure to simulate the evolution of the Newtonian drop differs slightly from algorithm 4.1 since use of the explicit algorithm 4.1 leads to impractically small time steps in the earlier stages of the evolution. Hence, we solve (3.18) iteratively at each time step by assuming a trial velocity field. The trial velocity field is used to calculate the stress tensor (2.9) and application of (3.18) yields the new velocity field, which serves as trial velocity field in the next iteration step. The iteration procedure is applied, until a self-consistent solution is obtained. To reduce the required number of iterations, we set the initial guess for the new velocity equal to the converged velocity at the previous time step.

In the next two subsections we will discuss the calculation of the velocity field and the time integration of both the non-Newtonian stress tensor and the boundary.

\subsection{EVALUATION OF THE INTEGRALS}

The numerical implementation of (3.18) requires the representation of the boundary $L$ into $N$ 'boundary elements' and a subdivision of the inner domain into $M$ 'internal cells'. The boundary elements used are circular arcs passing through three successive boundary points (Pozrikidis [20]). For the discretization of the inner domain we use triangles as shown in Fig. 3. The triangles in this figure result from a structured triangulation, in which the vertices have a polar distribution. Using this discretisation, we can write (3.18) as:

$$
\begin{array}{r}
\bar{u}_{\alpha}(\overline{\boldsymbol{x}})=\bar{u}_{\alpha}^{\infty}(\overline{\boldsymbol{x}})-\sum_{\ell=1}^{M} \int_{S_{\ell}^{(\phi)}} \bar{\tau}_{\beta \gamma}^{N N}\left(y, \rho_{y}\right) \bar{W}_{\alpha}^{\gamma \beta}\left(\overline{\boldsymbol{x}} ; y, \rho_{y}\right) \rho_{y} \mathrm{~d} \rho_{y} \mathrm{~d} y \\
-\sum_{\ell=1}^{N} \frac{1}{C} \int_{L_{\ell}} k(y) \bar{n}_{\beta}(y) \bar{M}_{\alpha}^{\beta}(\overline{\boldsymbol{x}} ; y) \rho_{y} \mathrm{~d} \ell_{y} .
\end{array}
$$

The next step is the actual numerical calculation of the integrals in Eq. (4.1). We distinguish two cases; (a) the boundary element or internal cell does not contain the point $\overline{\boldsymbol{x}}$ and (b) the boundary element or internal cell does contain the point $\bar{x}$. In case (a) the distance between a point $\bar{y}$ in the element and the point $\overline{\boldsymbol{x}}$ is always greater than zero, so that the singularities of 


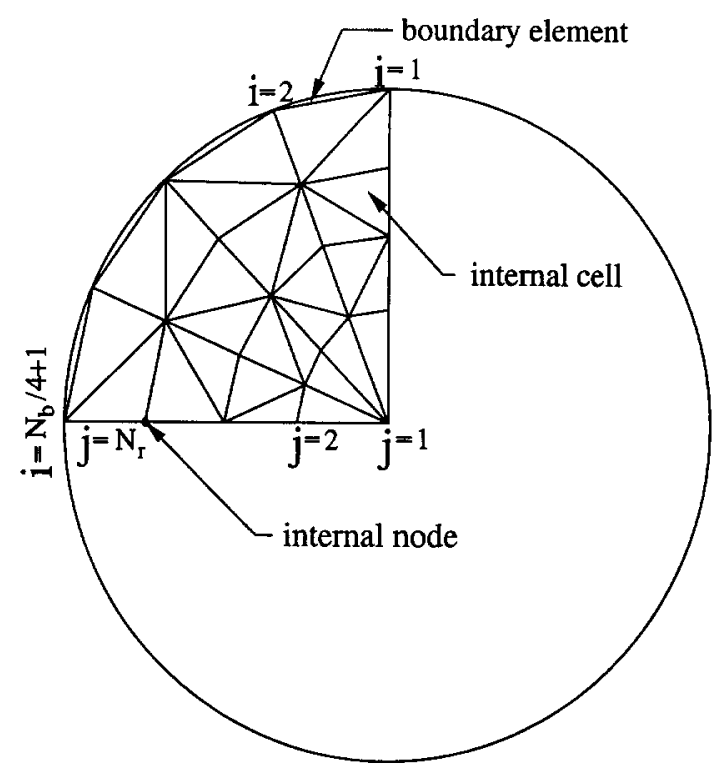

Fig. 3. The discretisation of the inner domain.

the kernels lie outside the integration domain. In this case we use a 16 point Gauss-Legendre quadrature for the boundary elements and a product rule based on a 4 point Gauss-Legendre quadrature for the domain elements (Patridge [21] and Evans [22]). In case (b) the distance between $\bar{x}$ and $\bar{y}$ can become zero and a special treatment of the singularities in the kernels is required. Analyzing the transformed Green's functions $\bar{M}_{\alpha}^{\beta}$ and $\bar{W}^{\gamma \beta}{ }_{\alpha}$ we find that both kernels are integrable. The first kernel has a logarithmic singularity, whereas the latter has a polar $(1 /|\overline{\boldsymbol{r}}|)$ singularity. To compute the boundary integral, we subtract the logarithmic singularity, and integrate analytically over the arc containing $\bar{x}$. The $1 /|\overline{\boldsymbol{r}}|$ singularity in the domain integral can be treated with the introduction of barycentrical coordinates $\xi_{1}, \xi_{2}, \xi_{3}$ over an internal cell with vertices $\bar{x}_{1}, \bar{x}_{2}$ and $\bar{x}_{3}$ (Toose [14]). Next the Green's function $\bar{W}_{\alpha}^{\gamma \beta}$ is written as the product of the polar singularity and a regular function $f_{\alpha \beta}^{\gamma \beta}$. Performing the integration over the internal cell and replacing $\xi_{2}$ by $\xi_{2}=\xi_{1} \xi_{2}^{*}$ we find that the domain integral yields:

$$
2 \Delta S_{\ell} \int_{\xi_{1}=0}^{1} \int_{\xi_{1}^{*}=0}^{1} \bar{\tau}_{\beta \gamma}^{N N}\left(y, \rho_{y}\right) \frac{1}{|\tilde{\boldsymbol{r}}|} \bar{f}_{\alpha}^{\gamma \beta}\left(\overline{\boldsymbol{x}} ; y, \rho_{y}\right) \rho_{y} \mathrm{~d} \xi_{2}^{*} \mathrm{~d} \xi_{1},
$$

with $\Delta S_{\ell}$ the area of the internal cell and $\tilde{r}=\left(\bar{x}_{1}-\bar{x}_{2}\right)-\left(\bar{x}_{2}-\bar{x}_{3}\right) \xi_{2}^{*}$. It is observed that the integral in (4.2) is regular, since the length of the vector $\tilde{r}$ is always greater than zero; hence a four-point Gauss-Legendre quadrature can be used to perform the integration over $\xi_{1}$ and $\xi_{2}^{*}$.

\subsection{TIME INTEGRATION}

In this subsection we describe a method to find the non-Newtonian stress tensor and the shape of the boundary $L$ at a new time level.

Updating the shape of the drop requires the calculation of the positions of all the boundary points on $L$ at the new time level. This calculation can be performed by time integration of 
(2.12) with an Euler forward scheme. When the grid points are moved in this way, both internal and boundary grid points will cluster and hence, gradually, a highly deformed grid results. The clustering arises directly from the fact that there are no restrictions on the stress tensor in a tangential direction, which implies that the points will move freely along the boundary in the direction of the external velocity field. We can reduce this clustering by moving the boundary nodes only in the direction normal to the boundary:

$$
\bar{x}_{\alpha}^{i N_{r}}\left(t_{n+1}\right)=\bar{x}_{\alpha}^{i N_{r}}\left(t_{n}\right)+\Delta t\left(\bar{u}^{i N_{r}} \cdot \bar{n}^{i}\right) \bar{n}_{\alpha}^{i}, i=1, \ldots, N_{b},
$$

where $N_{b}$ is the number of points along the boundary and $N_{r}$ the number of points in the radial direction, as shown in Fig. 3. The interior grid points $\left\{\bar{x}_{\alpha}^{i j}\right\}$ are found by interpolation between the center of the drop and the boundary points.

The new non-Newtonian stress tensor in algorithm 4.1 is obtained by integration of the constitutive equation. Using a partial or material time derivative we can evaluate the upperconvected time derivative in (2.7). The use of the partial time derivative, however, leads to a convective term which is somewhat difficult to calculate, since, owing to the deformation of the drop, a certain, fixed, point $\overline{\mathrm{x}}$, located inside the drop at a certain time $t$, may be outside the drop at the next time level. For this problem it is more convenient to use the material time derivative which does not require an explicit calculation of the convective term. This implies that the new non-Newtonian stress tensor $\tilde{\tau}_{\beta \gamma}^{N N}$ is defined on the grid, with nodes $\left\{\tilde{x}_{\alpha}^{i j}\right\}$, which is convected within the flow, i.e. on

$$
\tilde{x}_{\alpha}^{i j}\left(t_{n+1}\right)=\tilde{x}_{\alpha}^{i j}\left(t_{n}\right)+\Delta t \bar{u}_{\alpha}^{i j}, i=1 . . N_{b} \text { and } j=1, \ldots, N_{r} .
$$

Integration of (2.7) with an Euler forward scheme leads to:

$$
\bar{\tau}_{\beta \gamma}^{N N}\left(t_{n+1}\right)=\bar{\tau}_{\beta \gamma}^{N N}\left(t_{n}\right)+\Delta t\left(g^{\nu \mu}\left[\bar{\partial}_{\nu} \bar{u}_{\beta}\left(t_{n}\right) \bar{\tau}_{\mu \gamma}^{N N}\left(t_{n}\right)+\bar{\tau}_{\beta \nu}^{N N}\left(t_{n}\right) \bar{\partial}_{\mu} \bar{u}_{\gamma}\left(t_{n}\right)\right]+\bar{R}_{\beta \gamma}\left(t_{n}\right)\right) .
$$

with $t_{n}$ the discretized time defined as $t_{n}=n \Delta t$ with $\Delta t$ a constant time interval, $g^{\nu \mu}$ the metric tensor and $\bar{R}_{\beta \gamma}$ given by:

$$
\bar{R}_{\beta \gamma}\left(t_{n}\right)=\frac{\lambda^{(p)}}{D e}\left(\bar{\partial}_{\beta} \bar{u}_{\gamma}\left(t_{n}\right)+\bar{\partial}_{\gamma} \bar{u}_{\beta}\left(t_{n}\right)\right)-\frac{1}{D e} \bar{\tau}_{\beta \gamma}^{N N}\left(t_{n}\right)
$$

The covariant differentiation $\bar{\partial}_{\beta}$ in (4.5) and (4.6) is defined as (Bird [17]):

$$
\bar{\partial}_{\beta} \bar{u}_{\gamma}=\frac{\partial u_{\gamma}}{\partial \bar{x}^{\beta}}-\left\{\begin{array}{c}
\xi \\
\gamma \beta
\end{array}\right\} u_{\xi}
$$

where the coefficients $\left\{\begin{array}{c}\xi \\ \gamma \beta\end{array}\right\}$ are the Christoffel symbols. As an alternative a second- or higherorder Runge-Kutta scheme can be used. The partial derivatives in (4.5) and (4.6) are calculated with a finite-volume method (Van der Burg [23]).

Due to the Lagrangian approach, the new stress tensor $\bar{\tau}_{\beta \gamma}^{N N}\left(t_{n+1}\right)$ resulting from (4.5) is defined on the grid whose nodes $\left\{\tilde{x}_{\alpha}^{i j}\right\}$ are given by (4.4). In order to find it on the new grid nodes $\left\{\bar{x}_{\alpha}^{i j}\right\}$, the stress tensor is interpolated by:

$$
\tau_{\beta \gamma}^{N N}\left(\overline{\mathbf{x}}^{i j}\right) \approx \frac{1}{\sum 1 /\left(d^{i j}\right)^{2}} \sum \frac{\tau_{\beta \gamma}^{N N}\left(\tilde{\boldsymbol{x}}^{i j}\right)}{\left(d^{i j}\right)^{2}}
$$




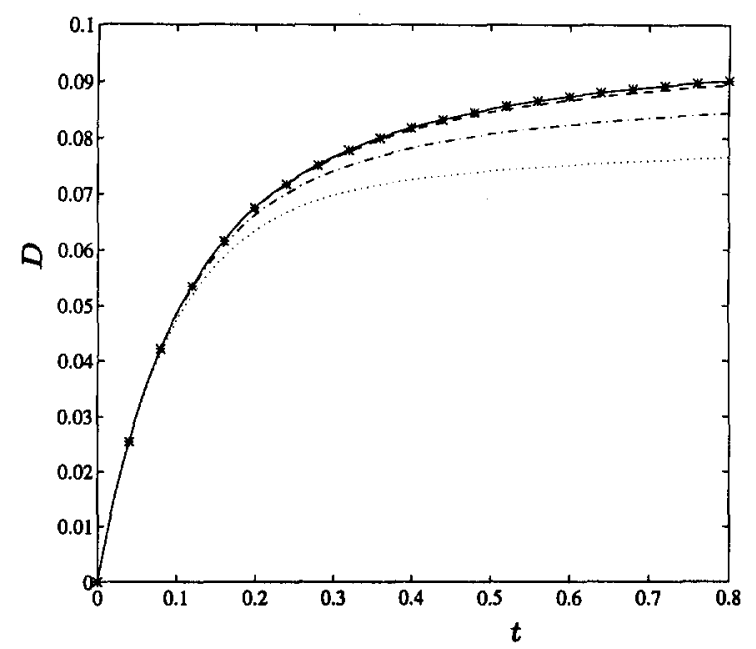

Fig. 4. The deformation of a drop at $C=0.05$ for five subsequent refinement levels. The dotted, dash-dotted, dashed and solid curves denote the deformation of a drop on a $12 \times 3,16 \times 5,24 \times 7$ and $32 \times 9$ grid respectively. The finest $48 \times 13$ grid is given by the asterisks.

with $d^{i j}=\left|\overline{\boldsymbol{x}}^{i j}-\tilde{\boldsymbol{x}}^{i j}\right|$ and the summation involves points up to the nearest neighbor. To ensure that this interpolation is sufficiently accurate, we introduce a time step restriction:

$$
\Delta t=\epsilon \min _{\ell}\left\{\frac{\Delta \bar{x}^{(\ell)}}{\bar{u}^{(\ell)}}\right\}
$$

where $\Delta \bar{x}^{(\ell)}$ is the shortest side of the $l$-th triangle, $\bar{u}^{(l)}$ the mean velocity over this triangle and $\epsilon$ is of the order of 0.1 .

\section{Results and discussion}

In this section we present the results of numerical calculations of the deformation of both Newtonian and non-Newtonian drops. In subsection 5.1 we verify numerically that the method used is mathematically correct and is second-order accurate in space. The order of accuracy in time corresponds to the time integration method applied. In subsection 5.2 the results for the Newtonian drop are compared with analytical results from literature. In subsection 5.3 we study the deformation of a drop filled with an Oldroyd-B fluid.

\subsection{VALIDATION OF THE NUMERICAL METHOD}

In the derivation of the integral representation for the velocity (2.13) we have assumed that the non-Newtonian stress tensor is continuous and has bounded derivatives. To show that the nonNewtonian stress tensor satisfies these conditions for all time, we performed a mathematical validation for an actual simulation of a two-dimensional drop (Toose [14]). The mathematical validation is based on the substitution of the numerical solution into the original equations (Toose [14]). The residual due to discretization errors should converge asymptotically to zero at a specific rate, as the numerically calculated quantities converge to the analytical solution in case the grid is refined. For the validation of the three-dimensional situation, we consider a drop subjected to an elongational flow at a capillary number of 0.05 . The Deborah number 


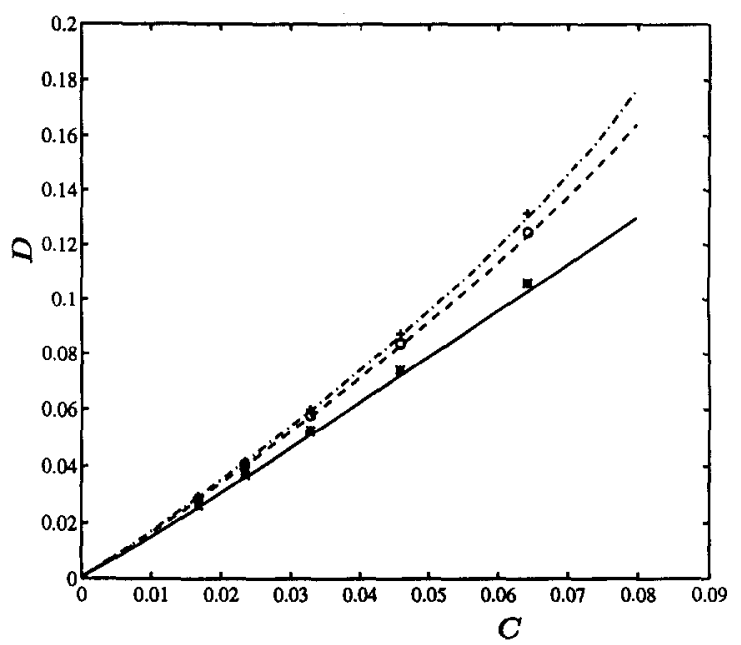

Fig. 5. Steady state deformation of a Newtonian drop for three values of $\lambda$. The solid, dashed and dash-dotted curves represent (5.2) at $\lambda=0, \lambda=1$, and $\lambda=2$ respectively. The asterisks, circles and crosses denote the numerical results at $\lambda=0,1$ and 2 respectively.

De and $\lambda^{(p)}$ are 0.25 and 0.1 respectively. Both the time step and the grid are refined with a Bulirsch-row. The computations were carried out with the first order-accurate Euler forward time-integration method. The residual, which was calculated at $t=0.096$ and $t=0.192$, converges to zero as a second-order process. Moreover, the deformation of the drop as a function of time must also converge as the grid is refined. In Fig. 4 we have plotted the deformation of a drop at a capillary number of 0.05 for five subsequent refinement levels. The time step is constant $(\Delta t=0.008)$ whereas the Deborah number $D e$ and $\lambda^{(p)}$ are the same as above. From this figure it can be seen that the deformation is converged sufficiently at the $32 \times 9$ grid. Hence, this grid and the $48 \times 13$ grid are used to perform the computations for the subsequent sections.

From these results we can conclude that the numerical solution satisfies the original equations and hence the smoothness assumptions made on the stress tensor are satisfied. The method has a second-order spatial accuracy when the mesh-size is decreased. The accuracy in time is first-order for the Euler and second-order for the compact storage four-stage RungeKutta scheme (Jameson [24]). In the next subsection it is shown that the method gives physically correct results for a Newtonian drop.

\subsection{DEFORMATION OF A NEWTONIAN DROP}

In this subsection we study the response of an axisymmetric Newtonian drop to an axisymmetric elongational flow and compare this with results from the literature. If the capillary number is sufficiently low, a drop placed in an elongational flow is known to deform, until a steady state is reached (Stone [2]). For small capillary numbers, Taylor [25] derived an expression relating the steady state deformation $\bar{D}$ to the corresponding capillary number. This expression is given by:

$$
\bar{D}=\frac{3}{2}\left(\frac{1+\frac{19}{16} \lambda}{1+\lambda}\right) C
$$




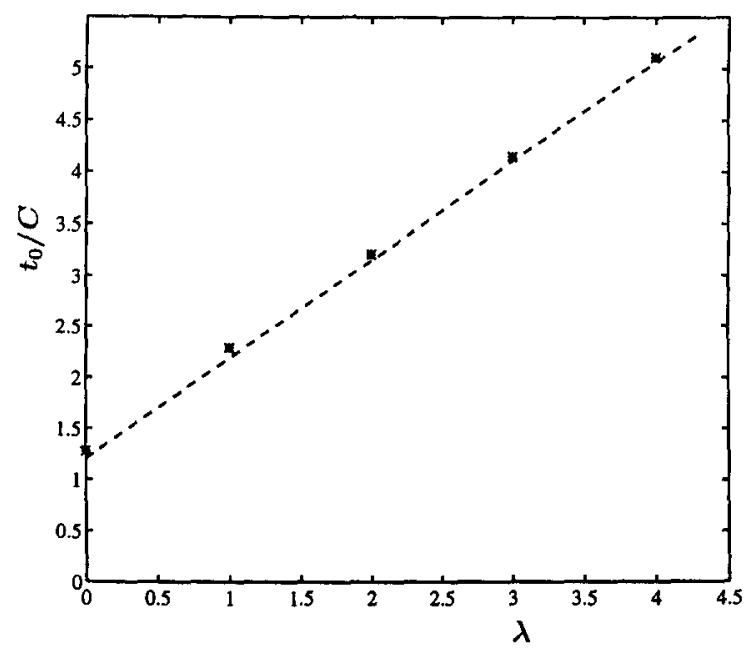

Fig. 6. The normalized relaxation time of a Newtonian drop at $C=0.05$ as a function of the viscosity ratio $\lambda$. The dashed line represents Eq. (5.4).

and is first-order accurate in $C$. Using the same technique as Taylor, Barthès-Biesel [26] derived an expression for $\bar{D}$ valid up to $O\left(C^{3}\right)$ which may be written in the form:

$$
\bar{D}=\frac{c_{0}(\lambda, C)}{1-c_{1}(\lambda, C)} C+O\left(C^{3}\right),
$$

in which $c_{0}(\lambda, C)$ and $c_{1}(\lambda, C)$ are complicated expressions which will not be given explicitly here. In Fig. 5 we have plotted expression (5.2) and some numerical results generated with algorithm 4.1 for three values of $\lambda$. We used a grid with 48 points along the boundary and 13 points in the radial direction. For small and moderate capillary numbers, the analytical and the numerical results are in very good agreement for all three values of $\lambda$.

For the time-dependent behavior of the Newtonian drop, analytical results valid to firstorder in the capillary number $C$ are known. For small capillary numbers the deformation of a drop in a general linear flow field can described by (Oldroyd [27] and Rallison [1]):

$$
D(t)=\bar{D}\left(1-e^{-t / t_{0}}\right),
$$

where the dimensionless relaxation time $t_{0}$ is given by:

$$
t_{0}=\frac{16+19 \lambda}{40(1+\lambda)}(3+2 \lambda) C \text {. }
$$

This behavior closely approximates actual simulations at sufficiently low capillary numbers. For the Newtonian drop, the relaxation time $t_{0}$ can be obtained by fitting the numerical results with the function given in (5.3). In Fig. 6 we plotted expression (5.4) and the numerically calculated relaxation times. The computations were performed on a $48 \times 13$ grid and a sufficiently small time step. The results indicate that the numerical computations are in good agreement with the analytical predictions.

From this we can conclude that the method gives correct results when the stress tensor is given by a Newtonian constitutive equation. In the next subsection we will concentrate on a drop which contains a non-Newtonian fluid. 


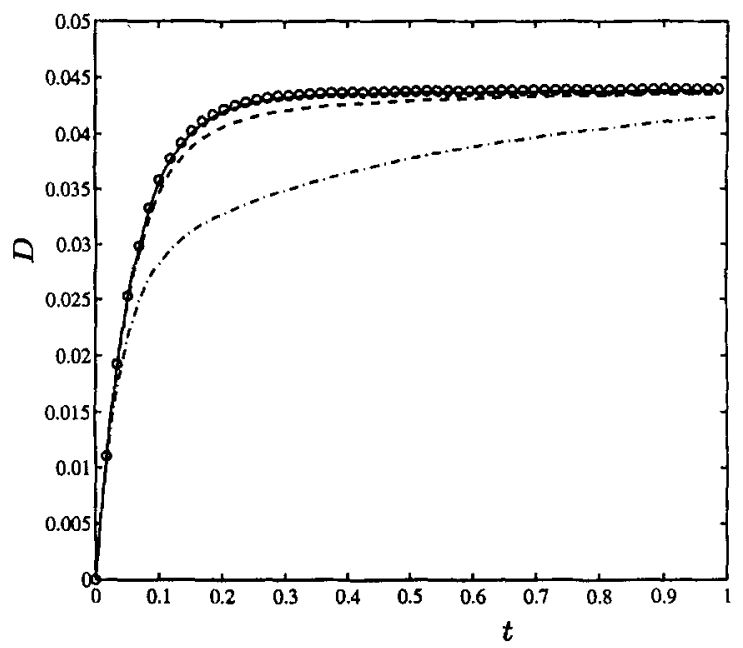

Fig. 7. Deformation of non-Newtonian drop at $C=0.025$ for a fixed Deborah number $D e=0.33$. The dash-dotted, dashed and solid lines are the responses of a non-Newtonian drop with $\lambda^{(p)}=6.7,0.89$ and 0.12 respectively. The circles show the response of the Newtonian drop at $\lambda=1$.

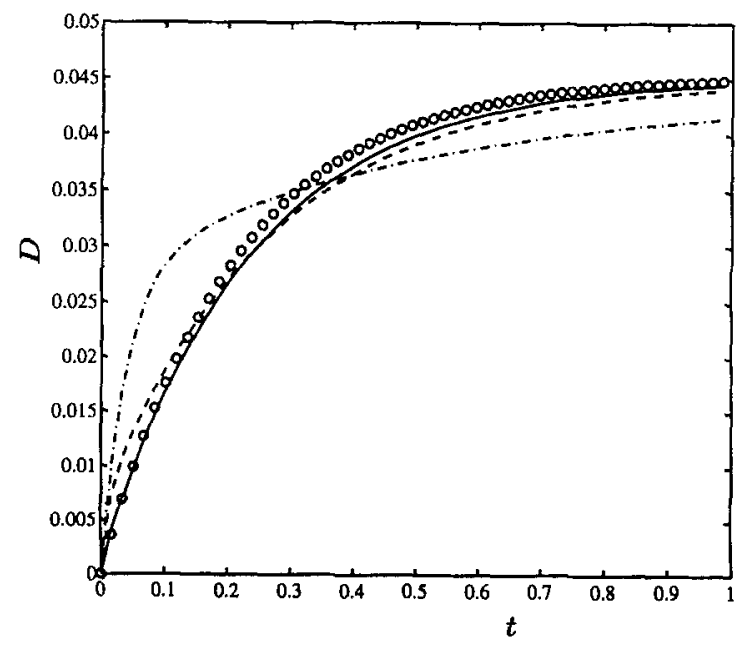

Fig. 8. Deformation of a non-Newtonian drop at $C=0.025$ for a fixed viscosity ratio $\lambda^{(p)}=0.33$. The dash-dotted, dashed and solid lines represent the response of a non-Newtonian drop with $D e=6.7,0.89$ and 0.12 respectively. The circles show the response of the Newtonian drop at $\lambda=1+\lambda^{(p)}$.

\subsection{DEFORMATION OF A DROP CONTAINING AN OLDROYD-B FLUID}

In this subsection we study the response of an axisymmetric drop containing an Oldroyd-B fluid in an elongational flow. For this non-Newtonian drop, no analytical or numerical results are known in the literature. To provide a way to verify our results, we investigate several limiting cases of the non-Newtonian stress tensor. Moreover, it is found that the response to a suddenly started elongational flow is governed by two characteristic relaxation times. In order to remove the effects of the deformation history of the cylinder, we use the relaxed state of the non-Newtonian stress tensor at $t=0$ (i.e. $Q=0$ ).

The Oldroyd-B model contains three independent parameters, the viscosity ratios $\lambda$ and $\lambda^{(p)}$ and the Deborah number $D e$. If either $\lambda^{(p)}$ or $D e$ approach zero, an essentially Newtonian 


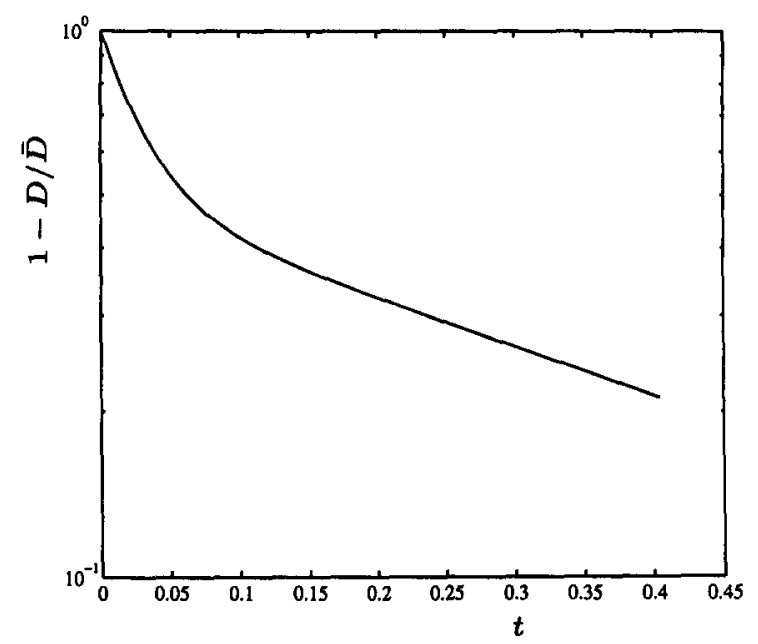

Fig. 9. Semi-logarithmic plot of the normalized deformation of a non-Newtonian drop at $C=0.025$. The Deborah number and viscosity ratio are given by 0.30 and 8.9 respectively.

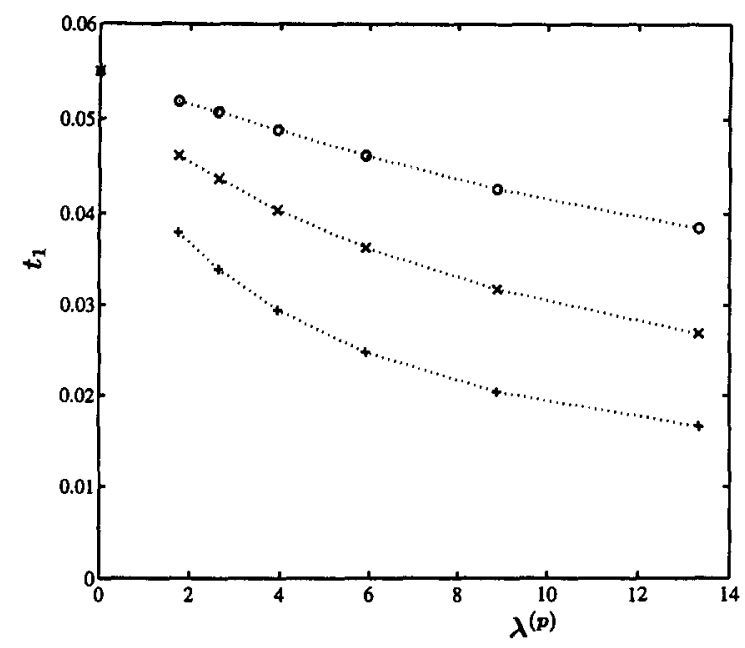

Fig. 10. The relaxation time $T_{1}$ of a non-Newtonian drop versus $\lambda^{(p)}$ at $C=0.025$ for a set of constant $D e$. The plusses, crosses and circles correspond to $D e=0.67, D e=0.30$ and $D e=0.13$ respectively. The asterisk at $\lambda^{(p)}=$ 0 gives the corresponding Newtonian limit.

behavior results. This will be used in the sequel to establish the correctness of the boundary integral method for the simulation of non-Newtonian drops. In the first case considered here $\lambda^{(p)}$ approaches zero, and the equation for the non-Newtonian stress tensor (2.7) reduces to:

$$
D_{e} \mathcal{D}_{t} \tau_{i j}^{N N}+\tau_{i j}^{N N}=0 .
$$

This equation implies that $\tau_{i j}^{N N}$ remains zero in time since $\tau_{i j}^{N N}=0$ at $t=0$. Hence the deformation should approach the Newtonian results as $\lambda^{(p)}$ tends to zero, which is demonstrated in Fig. 7. Here, the deformation of a drop for several values of $\lambda^{(p)}$ and constant $D e=0.33$ is plotted. The numerical results were obtained on a $32 \times 9$ grid with a time step of 0.001 . 
In the second case we let $D e$ approach zero, so that (2.7) reduces to:

$\tau_{i j}^{N N}=\lambda^{(p)} \dot{\gamma}_{i j}$

i.e. a Newtonian stress tensor. This implies that for $D e=0$ the non-Newtonian drop reduces to a Newtonian drop with a viscosity ratio $\lambda=1+\lambda^{(p)}$. In Fig. 8 the deformation of a drop for several values of $D e$ and constant $\eta^{(p)}=0.33$ is plotted. The numerical results were obtained with the same grid and time step as above. It is observed that for small De the deformation of the non-Newtonian drop approaches the deformation of the Newtonian drop with $\lambda=1+\lambda^{(p)}$. From this we can conclude that the limiting behavior of the Newtonian drop is recovered correctly. The behavior of the non-Newtonian drop for moderate values of $\lambda^{(p)}$ and $D e$ is surveyed next.

The results in Figs. 7 and 8 and further computations indicate that the main effect of variations in $\lambda^{(p)}$ and $D e$ for small capillary numbers is in the time- dependent behavior of the drop. From a semi-logarithmic plot of the normalized deformation $\left(D^{*}=1-D / \bar{D}\right)$ versus time it is observed that there are two dominant relaxation times (Fig. 9). So, in analogy with the Newtonian drop, we assume that for small capillary numbers the deformation can be described by:

$$
D=\bar{D}\left\{1-D_{1} e^{-t / t_{1}}-D_{2} e^{-t / t_{2}}\right\},
$$

i.e. we introduced an extra relaxation time accounting for the non-Newtonian behavior. The relaxation times $t_{1}$ and $t_{2}$ can be found by fitting the deformation to the expression given in (5.5). We obtain a close agreement between the simulation results and expression (5.5) for all values of $D e$ and $\lambda^{(p)}$. In Fig. 10 we have plotted the relaxation time $t_{1}$ showing the effects of variations in $\lambda^{(p)}$ at constant $D e$. It is seen that $t_{1}$ approaches the Newtonian limit $\left(t_{0}=0.055\right)$ as $\lambda^{(p)} \rightarrow 0$. The corresponding time $t_{2}$ tends to zero in this limit, indicating that the behaviour of the drop is dominated by only one relaxation time, i.e. the Newtonian response. We also studied the effect of variations in the Deborah number on the relaxation times $t_{1}$ and $t_{2}$. In this case the limiting behavior described before is also clearly displayed. In order to find the relaxation times for large $D e$ or small $\lambda^{(p)}$ we have to simulate the deformation of the drop for long periods of time using small time steps, since in this regime $t_{2} \gg t_{1}$ and we have to use $\Delta t \ll t_{1}$ in order to accurately capture the smallest relaxation time. The results and the physical interpretation of these computations will be published elsewhere.

From this we conclude that the limiting behavior of an Oldroyd-B drop is successfully recovered and that the deformation of the drop in time is governed by two relaxation times.

\section{Conclusions}

In this paper a boundary integral method for axisymmetric Newtonian and non-Newtonian drops immersed in a viscous fluid subjected to an axisymmetric flow has been presented. The non-Newtonian contribution was treated as a source term, leading to a domain integral in the boundary integral representation of the solution. To obtain a better connection with the matching conditions at the interface this domain integral was reformulated by applying Gauss' divergence theorem. In order to apply the divergence theorem it was necessary that the stress tensor be continuous and should have bounded derivatives for all times. These conditions were verified numerically in actual simulations and, hence, the reformulation of the domain integral was established. These simulations also show that the numerical method 
used is second order accurate in space. By transforming the integral representation for the velocity to cylindrical coordinates we were able reduce the dimension of the computational problem. The integral equation for the velocity kept the same form and the Green's functions were transformed explicitly to cylindrical coordinates.

The numerical results for the drop containing a Newtonian fluid have been compared with analytical results. The steady state deformation of a Newtonian drop was shown to be in agreement with analytical results (Taylor [25] and Barthès-Biesel [26]). The time-dependent behavior was compared with analytical results derived by Oldroyd [27]. For small capillary numbers the numerical and the analytical relaxation times were found to be in good agreement. For the drop containing an Oldroyd-B fluid, we examined the response to a suddenly started elongational flow. It was found that the behavior of the drop for small $D e$ and $\lambda^{(p)}$ approached the behavior of a Newtonian drop. The behavior of the drop for moderate values of $D e$ and $\lambda^{(p)}$ could be described by two relaxation times.

The Boundary Integral method developed in this paper is well suited for non-Newtonian drops, although computational times are much longer than in the Newtonian case, owing to the domain integral that appears in the formulation. The advantage of the method over a more direct (finite difference) discretization of the Stokes equations lies in the fact that only the drop has to be discretized and that relatively few points are needed to give accurate results. In the near future this method will be extended to so called vesicles in which the drop is formed by a Newtonian fluid which is encapsulated by a non-Newtonian lipid bilayer [11]. In this case, the advantages of the method can be fully exploited, since only a thin layer of non-Newtonian material is present and a correspondingly small part of the total flow domain has to be discretized. Moreover, in order to reduce the computational time, the possibility of evaluating the domain integral with an approximating boundary integral will be studied (Zheng [28]).

\section{Acknowledgement.}

This work was supported by the J.M. Burgers Center under EZ-project P-22. The authors wish to thank dr. R.M.J. van Damme and Prof. J. Mellema for several stimulating discussions. 


\section{Appendix}

In this appendix we give the components of the transformed Green's functions in the expression for the integral representation of the velocity (3.18).

The non-zero components of the function $\bar{M}_{\alpha}^{\beta}(\overline{\boldsymbol{x}} ; y)$ are given by:

$$
\begin{aligned}
\bar{M}_{1}^{1} & =E_{10}+\hat{x}^{2} E_{30} \\
\bar{M}_{2}^{1} & =\hat{x} \rho_{x} E_{30}-\hat{x} \rho_{y} E_{31} \\
\bar{M}_{1}^{2} & =-\hat{x} \rho_{y} E_{30}+\hat{x} \rho_{x} E_{31} \\
\bar{M}_{2}^{2} & =E 11-\rho_{x} r_{y} E_{30}+\left(\rho_{y}^{2}+\rho_{x}^{2}\right) E_{31}-\rho_{x} \rho_{y} E_{32} \\
\bar{M}_{3}^{3} & =\frac{\rho_{y} E_{11}+\rho_{x} \rho_{y}^{2} E_{30}-\rho_{x} \rho_{y}^{2} E_{32}}{\rho_{x}}
\end{aligned}
$$

where $\hat{x}=x-y$ and $E_{m n}$ given by:

$$
E_{m n}\left(\hat{x}, \rho_{x}, \rho_{y}\right)=\frac{4 k^{m}}{\left(4 \rho_{x} \rho_{y}\right)^{m / 2}} \int_{0}^{\pi / 2} \frac{\left(2 \cos ^{2} \phi-1\right)^{n}}{\left(1-k^{2} \cos ^{2} \phi\right)^{m / 2}} \mathrm{~d} \phi
$$

with

$$
k^{2}=4 \rho_{x} \rho_{y} /\left(\hat{x}^{2}+\left(\rho_{x}+\rho_{y}\right)^{2}\right) .
$$

The non-zero components of $\bar{W}_{\alpha}^{\gamma \beta}\left(\bar{x} ; y, \rho_{y}\right)$ are given by:

$$
\begin{aligned}
W_{11}^{11}= & -\bar{x} E_{30}+3 \bar{x}^{3} E_{50} \\
W_{1}^{12}= & -\rho_{x} E_{31}+3 \bar{x}^{2} \rho_{x} E_{51}-3 \bar{x}^{2} \rho_{y} E_{50}+\rho_{y} E_{30} \\
W_{1}^{21}= & \rho_{x} E_{31}+3 \hat{x}^{2} \rho_{x} E_{51}-3 \hat{x}^{2} \rho_{y} E_{50}-\rho_{y} E_{30} \\
W_{1}^{22}= & -6 \hat{x} \rho_{x} \rho_{y} E_{51}+3 \hat{x} \rho_{x}^{2} E_{52}+3 \hat{x} \rho_{y}^{2} E_{50}-\hat{x} E_{30} \\
W_{1}^{33}= & \left(-3 \hat{x} \rho_{x}^{2} E_{52}+3 \hat{x} \rho_{x}^{2} E_{50}-\hat{x} E_{30}\right) \rho_{y}^{2} \\
W^{11}= & \rho_{y} E_{31}-\rho_{x} E_{30}-3 \hat{x}^{2} \rho_{y} E_{51}+3 \hat{x}^{2} \rho_{x} E_{50} \\
W^{12}= & \hat{x} E_{31}+3 \hat{x}\left(\rho_{x}^{2}+\rho_{y}^{2}\right) E_{51}-3 \hat{x} \rho_{x} \rho_{y} E_{50}-3 \hat{x} \rho x \rho_{y} E_{52} \\
W^{21}= & -\hat{x} E_{31}+3 \hat{x}\left(\rho_{x}^{2}+\rho_{y}^{2}\right) E_{51}-3 \hat{x} \rho_{x} \rho_{y} E_{50}-3 \hat{x} \rho_{x} \rho_{y} E_{52} \\
W^{22}= & \rho_{y} E_{31}-\rho_{x} E_{30}-\left(3 \rho_{y}^{3}+6 \rho_{y} \rho_{x}^{2}\right) E_{51}+3 \rho_{x} \rho_{y}^{2} E_{50}+ \\
& \left(3 \rho_{x}^{3}+6 \rho_{x} \rho_{y}^{2}\right) E_{52}-3 \rho_{x}^{2} \rho_{y} E_{53} \\
W_{2}^{33}= & \left(\rho_{y} E_{31}-\rho_{x} E_{30}-3 \rho_{x}^{2} \rho_{y} E_{51}+3 \rho_{x}^{3} E_{50}-3 \rho_{x}^{3} E_{52}+3 \rho_{x}^{2} \rho_{y} E_{53}\right) / \rho_{y}^{2} \\
W^{13}= & \left(\hat{x} \rho_{x} E_{31}-3 \hat{x} \rho_{x}^{2} \rho_{y} E_{52}+3 \hat{x} \rho_{x}^{2} \rho_{y} E_{50}\right) / \rho_{y} \\
W^{23}= & \left(-\rho_{x} \rho_{y} E_{31}+3 \rho_{x}^{2} \rho_{y}^{2} E_{52}+\rho_{x}^{2} E_{30}+3 \rho_{x}^{3} \rho_{y} E_{51}-3 \rho_{x}^{2} \rho_{y}^{2} E_{50}-3 \rho_{x}^{3} \rho_{y} E_{53}\right) / \rho_{y} \\
W^{32}= & \left(-\hat{x} \rho_{x} E_{31}-3 \hat{x} \rho_{x}^{2} \rho_{y} E_{52}+3 \hat{x} \rho_{x}^{2} \rho_{y} E_{50}\right) / \rho_{y} \\
W^{33}= & \left(\rho_{x} \rho_{y} E_{31}+3 \rho_{x}^{2} \rho_{y}^{2} E_{52}-\rho_{x}^{2} E_{30}+3 \rho_{x}^{3} \rho_{y} E_{51}-3 \rho_{x}^{2} \rho_{y}^{2} E_{50}-3 \rho_{x}^{3} \rho_{y} E_{53}\right) / \rho_{y}
\end{aligned}
$$




\section{References}

1. J.M. Rallison, The deformation of small viscous drops and bubbles in shear flow. Ann. Rev. Fluid Mech. 16 (1984) 45-66.

2. H.A. Stone, Dynamics of drop deformation and breakup in viscous fluids. Ann. Rev. Fluid Mech. 26 (1994) 65-102.

3. O.A. Ladyzhenskaya, The Mathematical Theory of Viscous Incompressible Flow. New-York: Gordon and Breach (1969) $224 \mathrm{pp}$.

4. H.A. Lorentz, Eene algemeene stelling omtrent de beweging eener vloeistof met wrijving en eenige daaruit afgeleide gevolgen. Versl. K. Akad.W. Amsterdam 5 (1896) 168-175.

5. N.J. Nersessian and H.F. Cohen, Selected Works of H.A. Lorentz. Nieuwerkerk a/d IJssel: Palm publications (1987) $343 \mathrm{pp}$.

6. G.K. Youngren and A. Acrivos, Stokes flow past a particle of arbitrary shape: a numerical method of solution. J. Fluid Mech. 69 (1975) 377-403.

7. Q. Huang and T.A. Cruse, Some notes on singular integral techniques in boundary element analysis. Int.J. Num. Methods. Eng. 36 (1993) 2643-2659.

8. M. Tjahjadi, H.A. Stone and J.M. Ottino, Satellite and subsatellite formation in capillary breakup. J. Fluid Mech. 243 (1992) 297-317.

9. H.A. Stone and L.G. Leal, The effects of surfactants on drop deformation and breakup. J. Fluid Mech. 220 (1990) 161-186.

10. X.Z. Li, D. Barthès-Biesel and A. Helmy, Large deformations and burst of a capsule freely suspended in a elongational flow. J. Fluid Mech. 187 (1988) 179-196.

11. J.B.A.F. Smeulders, The Mechanical Properties of Lipid Bilayers. Ph. D. Thesis, University of Twente at Enschede (1992) 174 pp.

12. M.B. Bush, J.F. Milthorpe and R.I. Tanner, Finite element and boundary element methods for extrusion computations. J. Non-Newtonian Fluid Mech. 16 (1984) 37-51.

13. M.B. Bush, R.I. Tanner and P. Phan-Thien, A boundary element investigation of extrude swell. J. NonNewtonian Fluid Mech. 18 (1985) 143-162.

14. E.M. Toose, B.J. Geurts and J.M.G. Kuerten, A boundary integral method for two-dimensional (non)Newtonian drops in slow viscous flow. J. Non-Newtonian Fluid Mech., Accepted for publication.

15. R.G. Cox, The deformation of a drop in a general time-dependent fluid flow. J. Fluid Mech. 37 (1969) 601-623.

16. H.A. Lorentz, Abhandlungen über Theoretische Physic. Leibzig: Teubner (1907) $489 \mathrm{pp}$.

17. R.B. Bird, R.C. Amstrong and O. Hassager, Dynamics of Polymeric Liquids: Volume 1 Fluid Mechanics. New-York: Wiley-Interscience (1987) 649 pp.

18. P.F. Byrd and M.D. Friedman, Handbook of Elliptic Integrals for Engineers and Physicists. Berlin: SpringerVerlag (1954) $358 \mathrm{pp}$.

19. W.R. Schowalter, Mechanics of Non-Newtonian Fluids. Oxford: Pergamon Presss (1978) 300.

20. C. Pozrikidis, Boundary Integral and Singularity Methods for Linearized Viscous Flow. New-York: Cambridge University Press (1992) $256 \mathrm{pp}$.

21. P.W. Patridge, C.A. Brebbia and L.C. Wrobel, The Dual Reciprocity Boundary Element Method. Boston: Computational Mechanics Publications (1992) 276 pp.

22. G. Evans, Practical Numericalintegration. Guildford: Wiley (1993) 328 pp.

23. J.W. van der Burg, Numerical Techniques for Transonic Flow Calculations. Ph. D. Thesis, University of Twente at Enschede (1993) $171 \mathrm{pp}$.

24. A. Jameson, Transonic flow calculations. MAE-Report 1651, Princeton University (1983) $234 \mathrm{pp.}$

25. G.I. Taylor, The viscosity of a fluid containing small drops of another fluid. Proc. R. Soc. London. A146 (1932) 41-48.

26. D. Barthès-Biesel, Deformation and burst of a liquid droplet freely suspended in a linear shear field. J. Fluid Mech. 61 (1973) 1-21.

27. J. G. Oldroyd, The elastic and viscous properties of emulsions and suspensions. Proc. R. Soc. London. A218 (1953) 122-132.

28. R. Zheng, N. Phan-Thien and C.J. Coleman, A boundary element approach for non-linear boundary-value problems. Computational Mech. 8 (1991) 71-86. 